\title{
Chemogenetic Activation of Midbrain Dopamine Neurons Affects Attention, but not Impulsivity, in the Five-Choice Serial Reaction Time Task in Rats
}

\author{
Linde Boekhoudt', , Elisa S Voets', Jacques P Flores-Dourojeanni' ${ }^{1,2}$, Mieneke CM Luijendijk', \\ Louk JMJ Vanderschuren*,2 and Roger AH Adan*,I \\ 'Brain Center Rudolf Magnus, Department of Translational Neuroscience, University Medical Center Utrecht, Utrecht, The Netherlands; \\ ${ }^{2}$ Department of Animals in Science and Society, Division of Behavioural Neuroscience, Faculty of Veterinary Medicine, Utrecht University, Utrecht, \\ The Netherlands
}

\begin{abstract}
Attentional impairments and exaggerated impulsivity are key features of psychiatric disorders, such as attention-deficit/hyperactivity disorder, schizophrenia, and addiction. These deficits in attentional performance and impulsive behaviors have been associated with aberrant dopamine (DA) signaling, but it remains unknown whether these deficits result from enhanced DA neuronal activity in the midbrain. Here, we took a novel approach by testing the impact of chemogenetically activating DA neurons in the ventral tegmental area (VTA) or substantia nigra pars compacta (SNc) on attention and impulsivity in the five-choice serial reaction time task (5-CSRTT) in rats. We found that activation of DA neurons in both the VTA and SNc impaired attention by increasing trial omissions. In addition, SNc DA neuron activation decreased attentional accuracy. Surprisingly, enhanced DA neuron activity did not affect impulsive action in this task. These results show that enhanced midbrain DA neuronal activity induces deficits in attentional performance, but not impulsivity. Furthermore, DA neurons in the VTA and SNc have different roles in regulating attention. These findings contribute to our understanding of the neural substrates underlying attention deficits and impulsivity, and provide valuable insights to improve treatment of these symptoms. Neuropsychopharmacology (2017) 42, I3I5-1325; doi:I0.1038/npp.2016.235; published online 16 November 2016
\end{abstract}

\section{INTRODUCTION}

Attentional deficits and exaggerated impulsivity are core features of several psychiatric disorders, including attentiondeficit/hyperactivity disorder, schizophrenia, and addiction (American Psychiatric Association, 2013). The brain dopamine (DA) system is known to be involved in the psychopathology of these disorders (Everitt and Robbins, 2005; Franken et al, 2005; Howes and Kapur, 2009; Tripp and Wickens, 2009; Volkow and Baler, 2015), as well as in the regulation of attention and impulsivity (Bari and Robbins, 2013; Cools, 2011; Nieoullon, 2002; Pattij and Vanderschuren, 2008). However, the relationship between DA signaling, attention, and impulse control remains incompletely understood. Previous research has mainly focused on DA signaling in target areas such as the striatum and prefrontal cortex (PFC). DA signaling in these areas depends on DA neuronal activity in the midbrain, but it is

\footnotetext{
*Correspondence: L Boekhoudt or Professor LJMJ Vanderschuren or Professor RAH Adan, Brain Center Rudolf Magnus, Department Translational Neuroscience, University Medical Center Utrecht, Universiteitsweg 100, Utrecht 3584CG, The Netherlands, Tel: +31 8875 68877, E-mail: lindeboekhoudt@gmail.com or l.j.m.j.vanderschuren@uu.nl or r.a.h.adan@umcutrecht.nl

Received 25 May 2016; revised 30 September 2016; accepted 4 October 2016; accepted article preview online 17 October 2016
}

unknown whether abnormal activity of DA neurons underlies attentional deficits and/or impulsivity.

Here, we took a novel approach by testing the impact of chemogenetic activation of midbrain DA neurons on attentional performance and impulsive behavior in rats. We used designer receptors exclusively activated by designer drugs (DREADD; Armbruster et al, 2007) in TH::Cre transgenic rats, to selectively increase DA neuronal activity in either the ventral tegmental area (VTA) or substantia nigra pars compacta $(\mathrm{SNc})$. The effects on attention and impulsivity were tested in the five-choice serial reaction time task (5-CSRTT; Carli et al, 1983). In this task, the rats are trained to respond to a visual stimulus, which is briefly and pseudo-randomly presented in one of five nose-poke holes. Impulsivity in the 5-CSRTT is measured by assessing premature responses that are made before the stimulus onset. Elevated DA signaling in the striatum, especially the nucleus accumbens (NAc) and dorsomedial striatum (DMS), has been associated with premature responding (Agnoli et al, 2013; Baarendse and Vanderschuren, 2012; Economidou et al, 2012; Van Gaalen et al, 2006; Moreno et al, 2013; Pattij et al, 2007; Pezze et al, 2007). Attentional performance is tested by assessing response accuracy and trial omissions, which are mainly affected by DAergic manipulations in the PFC and dorsal striatum (Agnoli and Carli, 2011; Agnoli et al, 2013; Baunez and Robbins, 1999; Economidou et al, 
2012; Granon et al, 2000; Rogers et al, 2001; Winstanley et al, 2010). These findings suggest a role for both the mesocorticolimbic and nigrostriatal DA pathway-ascending from DA neurons in VTA and SNc, respectively (Bjorklund and Dunnett, 2007; Moore and Bloom, 1978)-in the regulation of attention and impulsivity. In this study, we tested whether enhanced midbrain DA neuronal activity is sufficient to induce attentional deficits and/or impulsive actions in rats. As pharmacological stimulation of DA signaling, via DA receptor (DA-R) agonists or DA reuptake inhibition, has repeatedly been shown to promote impulsive actions (Agnoli et al, 2013; Baarendse and Vanderschuren, 2012; Economidou et al, 2012; Van Gaalen et al, 2006; Moreno et al, 2013; Pattij et al, 2007; Pezze et al, 2007), we hypothesized that chemogenetic activation of midbrain DA neurons would increase premature responding. Given the major role of the NAc and DMS in impulsivity (Dalley et al, 2011; Eagle and Baunez, 2010), we predicted that particularly VTA DA neuron activation would enhance impulsive actions, whereas SNc DA neuron activation would have a more modest effect. Attentional performance in the 5CSRTT has been shown to either be improved or impaired following the administration of DA-R agonists and DA reuptake inhibition (Agnoli et al, 2013; Baarendse and Vanderschuren, 2012; Besson et al, 2010; Economidou et al, 2012; Granon et al, 2000; Pezze et al, 2007; Winstanley et al, 2010). Therefore, we hypothesized that activation of DA neurons in either VTA or SNc would affect attentional accuracy and trial omissions, although the directionality of the effects was not readily predictable.

\section{MATERIALS AND METHODS}

\section{Animals}

Male TH::Cre ${ }^{+/-}$rats were bred in-house, by crossing heterozygous $\mathrm{TH}: \mathrm{Cre}^{+/-}$rats with wild-type Long Evans mates. In total, 42 rats were used, divided into three groups: (1) VTA:Dq+, $n=12 \mathrm{Cre}^{+/-}$rats, with DREADD in the VTA; (2) SN:Dq+, $n=16 \mathrm{Cre}^{+/-}$rats with DREADD in the $\mathrm{SNc}$; and (3) Cre,$- n=14$, a control group of $\mathrm{Cre}^{-l-}$ littermates, which received DREADD virus in the VTA, but do not express Cre and thus do not express DREADD. In each behavioral test, the effects of clozapine-N-oxide (CNO) were compared with saline control treatment in each animal. A Cre-negative group was included to control for potential effects of CNO administration in the absence of DREADD expression. To reduce the number of animals used in this study, we have included a single Cre-negative control group, infused with Cre-dependent DREADD virus in the VTA. Earlier (pilot) studies have shown that infusion of DREADD virus into the SNc in Cre-negative rats did not result in any DREADD expression, nor did we observe any behavioral effects of CNO administration. The rats were socially housed in Macrolon type III or IV cages, in a temperature- and humidity-controlled room. A wood block was provided for cage enrichment. The rats were housed under a reversed $12 \mathrm{~h}$ day-night cycle (lights on at 19:00), allowing behavioral training and testing during the animals' active phase. The rats had ad libitum access to water. Throughout the experiment, the rats were food restricted to maintain $90 \%$ of their free-feeding body weight. All the experiments were approved by the Animal Ethics Committee of Utrecht University and were conducted in accordance with Dutch laws (Herziene Wet op Dierproeven, Art 10.a.2, 2014) and European regulations (Guidelines 86/609/EEC and 2010/63/EU).

\section{Surgery}

All the animals were injected bilaterally with $1 \mu \mathrm{l}$ of AAV5hSyn-DIO-hM3Dq-mCherry $(6.4-8.0 \times$ E12 molecules $/ \mathrm{ml}$; UNC Vector Core), using a stereotactic apparatus. The surgery was performed in two cohorts, one before operant training ( $n=22,7$ weeks old, $\sim 200 \mathrm{~g}$ ), and one after training ( $n=20,27$ weeks old, $\sim 450$ gram). For the first cohort, coordinates for VTA were set at AP: $-5.4, \mathrm{ML}:+1.3$ ( $5^{\circ}$ angle), DV: $-8.0 \mathrm{~mm}$; and $\mathrm{SNc}$ at AP: $-5.2, \mathrm{ML}:+2.0$, DV: -7.2 . The second cohort received VTA injections at AP: $-5.8, \mathrm{ML}:+1.3$ ( $5^{\circ}$ angle), DV: -8.4 ; and $\mathrm{SNc}$ at $\mathrm{AP}-5.4, \mathrm{ML}+2.2, \mathrm{DV}-7.7$. All the coordinates are in millimeters relative to Bregma. The virus was infused at a rate of $0.2 \mu \mathrm{l} / \mathrm{min}$ for $5 \mathrm{~min}$, and the needle was left in place for $10 \mathrm{~min}$ after infusion. The surgery was performed under anesthesia, induced by intramuscular fentanyl/fluanisone $(0.315 \mathrm{mg} / \mathrm{kg}$ fentanyl, $10 \mathrm{mg} / \mathrm{kg}$ fluanisone; Hypnorm, Janssen Pharmaceutical). Local anesthesia was provided by xylocaine, sprayed on the skull (Lidocaine $100 \mathrm{mg} / \mathrm{ml}$, AstraZeneca BV). Carprofen $(5.0 \mathrm{mg} / \mathrm{kg}$, subcutaneously, Carporal, AST Farma BV) was given for pain relief, at the day of surgery and the two following days. The rats were housed individually for 1 week under DM-II conditions, to recover from surgery. There were at least 8 weeks in between surgery and behavioral testing.

\section{Five-Choice Serial Reaction Time Task}

Behavioral training and testing on the 5-CSRTT were performed as previously published (Baarendse and Vanderschuren, 2012), with slight adjustments. All the training and testing took place in operant conditioning chambers $(30.5 \times 24 \times 21 \mathrm{~cm}$, Med-Associates, USA $)$, placed in sound attenuated boxes, and controlled by MED-PC version IV (Med-Associates). The chambers were equipped with five evenly spaced nose-poke holes in a curved wall, each with a yellow light-emitting diode stimulus light and an infrared detector, and a food magazine for pellet delivery in the opposite wall. Following habituation to the setup, the rats were trained to respond to an illuminated nose-poke hole, which was rewarded with delivery of a sucrose pellet (45 mg, TestDiet, USA) in the food magazine. Over six different training stages, the rats learned to perform this task under baseline conditions of $5 \mathrm{~s}$ intertrial interval (ITI), $1 \mathrm{~s}$ stimulus duration (SD) and $5 \mathrm{~s}$ limited hold.

All the sessions started with the delivery of a free sucrose pellet into the food magazine, after which the rat could initiate a trial by nose-poking into the food magazine. Each trial started with a $5 \mathrm{~s}$ ITI, before a stimulus light was presented in one of the five nose-poke holes, in a pseudorandom order. When a correct response was made, ie, a nose-poke in the illuminated hole within the limited hold period, a sucrose pellet was delivered. A response in one of the other four holes was recorded as an incorrect response, a failure to respond within the limited hold period as an 
omission, and a nose-poke made during the ITI as a premature response. Incorrect responses, omissions, and premature responses were punished with a $5 \mathrm{~s}$ time-out, during which stimulus lights were switched off and the house light was switched on. Repeated responses in one of the nosepoke holes were recorded as perseverative responses, but had no programmed consequences. Latencies to respond (premature, correct, incorrect, and food magazine entry) were recorded as well. The next trial started either when the animal entered the food magazine to collect the reward, or automatically after a time-out. The sessions lasted for 100 trials or $30 \mathrm{~min}$, whichever occurred first. For analysis, omissions were defined as the percentage of omitted trials relative to total number of trials (omissions/[correct+incorrect+omissions] $\times 100 \%)$. The number of omissions following either a correct response or a time-out were calculated from trial-by-trial data, and analyzed as percentage of total omissions, to correct for any changes in total number of omitted trials. Accuracy was defined as percentage of correct responses relative to total responses (correct/ [correct+incorrect] $\times 100 \%)$. Other parameters were analyzed as absolute values.

Pharmacological testing started after approximately 3 months, when the rats showed stable baseline performance levels. Task performance was tested $30 \mathrm{~min}$ after administration of selective DREADD ligand $\mathrm{CNO}$ or saline, using a within-subject, counter-balanced design. Challenge sessions were separated by at least two baseline training sessions (5 s ITI, $1 \mathrm{~s}$ SD). Dose-response testing for $\mathrm{CNO}$ was conducted using a Latin Square design, with at least one wash-out baseline day between test sessions.

\section{Drugs}

CNO (kindly provided by Bryan Roth (University of North Carolina, Chapel Hill NC, USA)) and the NIMH Chemical Synthesis and Drug Supply Program) and d-amphetamine sulphate (OPG Utrecht, The Netherlands) were dissolved in sterile $0.9 \%$ saline and kept at $4{ }^{\circ} \mathrm{C}$ in between testing. All the injections were given intraperitoneally, at $1 \mathrm{ml} / \mathrm{kg}$ bodyweight. CNO was given at a dose of $0.3 \mathrm{mg} / \mathrm{kg}$, unless stated otherwise. This dose was chosen based on previous work, in which we showed that $0.3 \mathrm{mg} / \mathrm{kg}$ yielded significant behavioral effects (ie, increased locomotor activity) in rats expressing hM3Dq in DA neurons in either VTA or SNc (Boekhoudt et al, 2016).

\section{Tissue Preparation and Immunohistochemistry}

Killing, tissue preparation, and immunohistochemical analysis were performed as previously described (Boender et al, 2014). The rats received a lethal dose of sodium pentobarbital $(0.1 \mathrm{ml} / 100 \mathrm{~g}$ bodyweight; Euthanimal, Alfasan BV, The Netherlands), and were transcardially perfused with $0.9 \%$ saline followed by $4 \%$ paraformaldehyde (PFA) in phosphate-buffered saline (PBS). The brain slices $(40 \mu \mathrm{m})$ were stained for tyrosine hydroxylase (TH) and hM3DqmCherry with Mouse anti-TH (EMD Millipore, 1:500) and Rabbit anti-dsRed (Clontech, $1: 500$ ), respectively. Overnight incubation (at $4{ }^{\circ} \mathrm{C}$ ) with these primary antibodies was followed by $2 \mathrm{~h}$ incubation with secondary antibodies Goat anti-Rabbit $568 \quad(1: 500)$ and Goat anti-Mouse 488
(Molecular Probes, 1:1000). All the antibodies were dissolved in PBS containing 0.05\% Tween. The slices were mounted with FluorSave (EMD Millipore).

\section{Quantification of DREADD Expression}

Fluorescent pictures were taken using a Zeiss Axio Scope A1 microscope and AxioVision software, with identical settings for all animals. Intensity of hM3Dq-mCherry fluorescence in midbrain and striatum was analyzed using ImageJ. Fluorescence intensity (0-255, arbitrary units) was measured at selected sub-regions in the midbrain (VTA and dorsal part of $\mathrm{SNc}$ (SNcd) -5.2 to $-6.0 \mathrm{~mm}$ from Bregma; medial part of $\mathrm{SNc}(\mathrm{SNm})-5.6$ to $-6.0 \mathrm{~mm}$ from Bregma) and striatum (NAc Core, NAc Shell, DMS, dorsolateral striatum (DLS), 2.0 to $1.0 \mathrm{~mm}$ from Bregma). mCherry fluorescence was not detectable in other DAergic target regions, including the PFC. Background fluorescence (measured at periacquaductal grey for midbrain and insular cortex for striatum) was subtracted from the measurements, and average fluorescence intensity was calculated per animal (two to eight measurements per region).

\section{Data Analysis}

Fluorescence levels per region were compared between groups using Newman-Keuls test, followed by post hoc Mann-Whitney $U$-tests ( $P$-value corrected for multiple comparisons). In total, 6-10 rats were included for the Cre - control group and 13-15 rats for VTA:Dq+ and $\mathrm{SN}: \mathrm{Dq}+$ groups.

No animals were excluded from the analysis on the basis of DREADD expression. For behavioral analysis, the rats were included based on baseline performance criteria $(\leqslant 20 \%$ omissions and $\geqslant 85 \%$ accuracy). One rat in the $\mathrm{SN}: \mathrm{Dq}+$ group did not meet these criteria and was excluded from all the analyses. Furthermore, the animals were excluded from analysis of specific tests when baseline performance before drug administration did not meet the selection criteria. In total, 12-15 animals per group were included for each behavioral analysis. Effects of CNO compared with saline were tested using repeated-measures general linear model (RM-GLM). When RM-GLM indicated a significant interaction between experimental Group (VTA:Dq+/SN:Dq $+/ \mathrm{Cre}-$ ) and Treatment (CNO/saline), post hoc pairwise comparisons were performed per group. Exploratory studies for the effects of amphetamine or a $7 \mathrm{~s}$ ITI were analyzed by paired samples $t$-tests. Effects of challenges compared with baseline conditions were tested with RM-GLM, using only data from saline test days, and post hoc tests were performed following either a significant main effect of Challenge, or Challenge $\times$ Group interaction. Dose-response analysis of CNO was tested with RM-GLM. On the basis of Mauchly's test of sphericity, degrees of freedom were adjusted according to Huyn-Feldt correction (when Huyn-Feldt epsilon $\geqslant 0.7$ ) or Greenhouse-Geisser (when Huyn-Feldt epsilon <0.7). Statistical significance was set at $\alpha=0.05$, and Bonferroni corrections were made for multiple comparisons. Statistical tests were performed using SPSS 16.0 and graphs were made using GraphPad Prism 6 and Adobe Illustrator. 
a

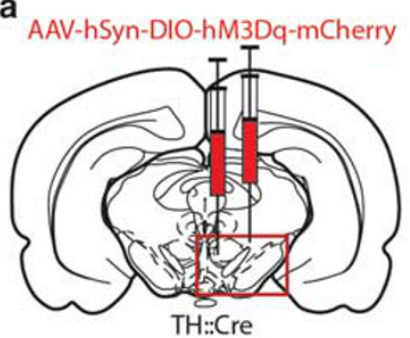

VTA:Dq+
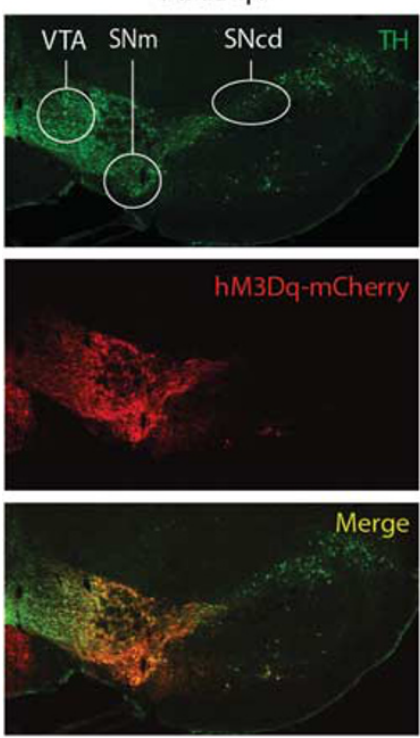

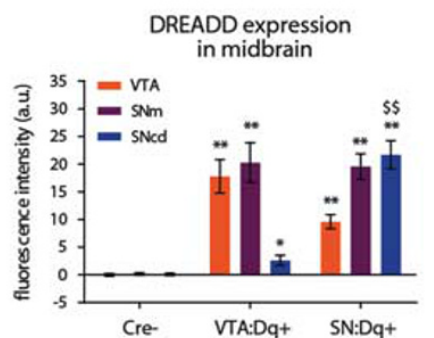

$\mathrm{SN}: \mathrm{Dq}+$
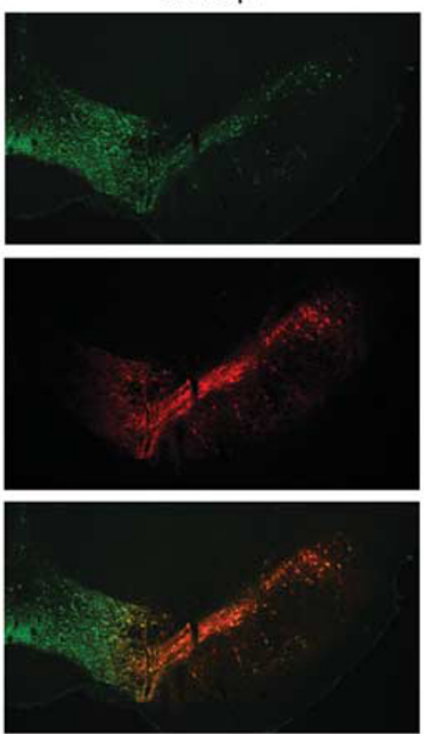

b
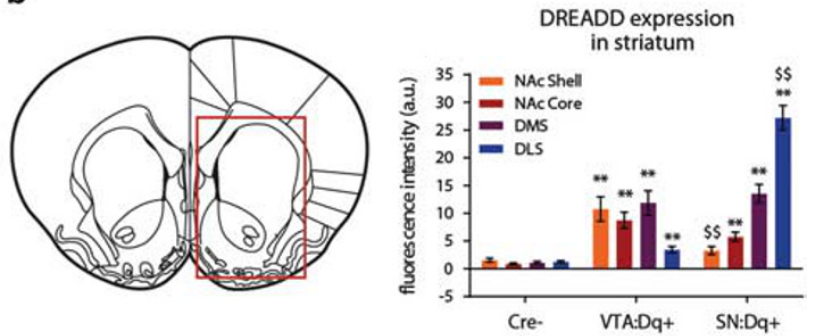

VTA:Dq+
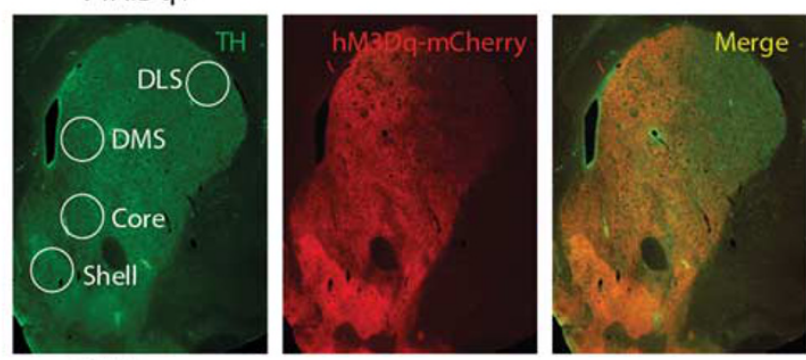

$\mathrm{SN}: \mathrm{Dq}+$
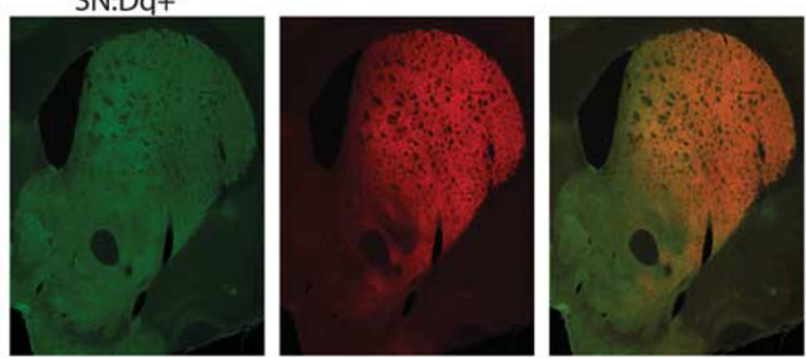

Figure I DREADD expression in DA neurons in VTA or SNc. DREADD expression in midbrain (a) and striatal projection areas (b). All the rats received bilateral injection with Cre-dependent DREADD virus into VTA or SNc (a, top left). Red rectangle represents area depicted in lower panels, showing representative examples of expression of tyrosine hydroxylase $(\mathrm{TH})$ and DREADD (hM3Dq-mCherry) in midbrain and striatum of TH::Cre rats expressing DREADD in the VTA (VTA:Dq+) or SNc (SN:Dq+). Top right panels show quantified fluorescence levels in selected sub-regions in the midbrain and striatum. Error bars represent mean $\pm \mathrm{SEM} * P<0.05$, ** $P<0.0$ I difference compared with Cre - control group. ${ }^{\$} P<<0.0$ I difference between $\mathrm{VTA}: \mathrm{Dq}+$ and $\mathrm{SN}: \mathrm{Dq}+$ groups.

\section{RESULTS}

\section{DREADD Expression}

All the rats were injected with Cre-dependent DREADD virus (AAV-hSyn-DIO-hM3Dq-mCherry) in either the VTA or SNc (Figure 1a). Immunohistochemical analysis confirmed DREADD expression in the VTA in VTA:Dq+ rats, and in SNc in SN:Dq+ rats. (Figure 1a). To quantify expression levels in midbrain as well as striatum, mCherry fluorescence intensity was measured in specific sub-regions (Figure 1a and $\mathrm{b}$ ). This revealed that the VTA:Dq+ group primarily expressed DREADD in the VTA and SNm (fluorescence levels both $P<0.0015$ compared with Cre -), and also showed modest expression in SNcd $(P=0.015$ compared with Cre-; Figure 1a). This was reflected in the striatum by DREADD expression primarily in NAc Core and Shell, as well as DMS, and to a lesser extent DLS (Figure 1b; all $P<0.01$ compared with Cre - ). In contrast, the $\mathrm{SN}: \mathrm{Dq}+$ group primarily showed DREADD expression in SNm and SNcd, as well as modest expression in VTA (all $P<0.0015$ compared with $\mathrm{Cre}-$ ). In the striatum, this was reflected by expression primarily in DLS, as well as DMS and NAc Core (Figure 1b). Compared with the VTA:Dq+ group, the
$\mathrm{SN}: \mathrm{Dq}+$ group showed significantly higher expression levels in SNcd and DLS, but lower expression in NAc Shell (Figure 1a and b). Thus, VTA:Dq+ rats mainly showed DREADD expression in the mesolimbic pathway, including projections to NAc and DMS, whereas $\mathrm{SN}: \mathrm{Dq}+$ rats mainly showed expression in the nigrostriatal pathway, towards dorsal striatum.

The rats operated in the second cohort showed higher intensity of DREADD expression compared with the first cohort, which is likely due to the use of a different virus batch. As behavioral effects of CNO did not differ between cohorts, the results were pooled for statistical analysis.

\section{Effects of DA Neuron Activation on Attention}

Chemogenetic activation of DA neurons impaired attentional performance in the 5-CSRTT under baseline conditions. In both VTA:Dq+ and $\mathrm{SN}: \mathrm{Dq}+$ rats, omissions were increased after CNO treatment (Figure 2a; Group $\times$ Treatment interaction $\mathrm{F}_{2,37}=5.4, P=0.009$; post hoc VTA:Dq+ and $\mathrm{SN}: \mathrm{Dq}+$, both $P<0.0005)$. Furthermore, $\mathrm{CNO}$ treatment decreased accuracy in the $\mathrm{SN}: \mathrm{Dq}+$ group (Figure 2b; Group $\times$ Treatment interaction $\mathrm{F}_{2,37}=10.432, \quad P<0.0005$; 


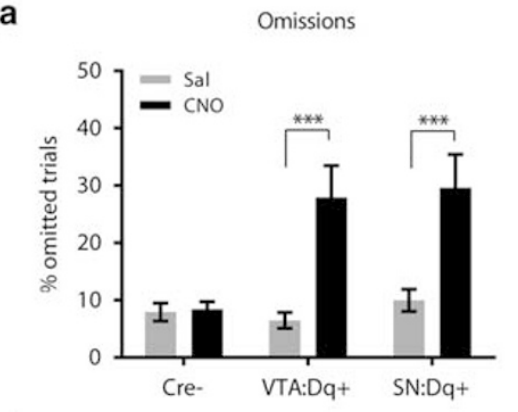

d
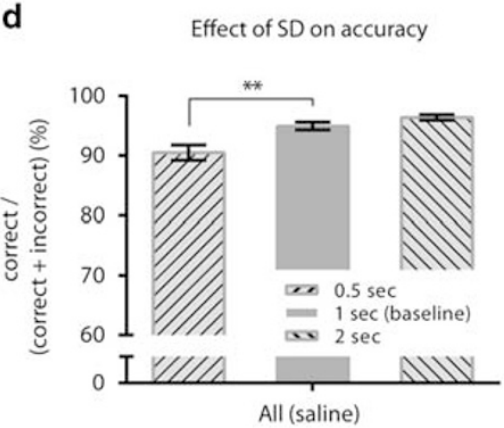

e

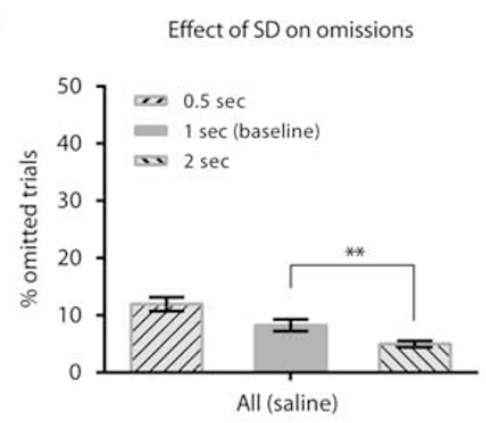

b

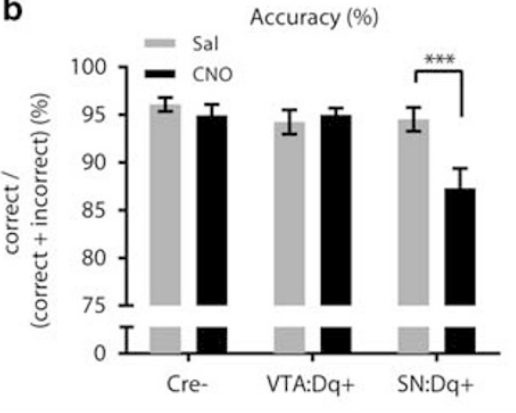

C

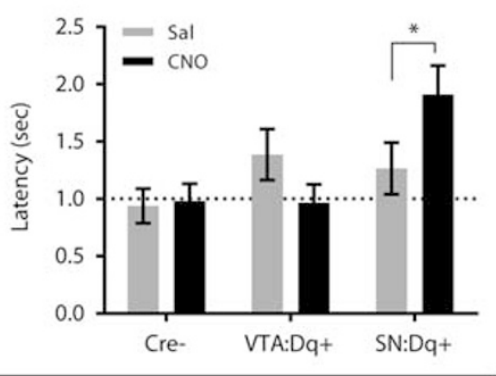

Latency to incorrect response
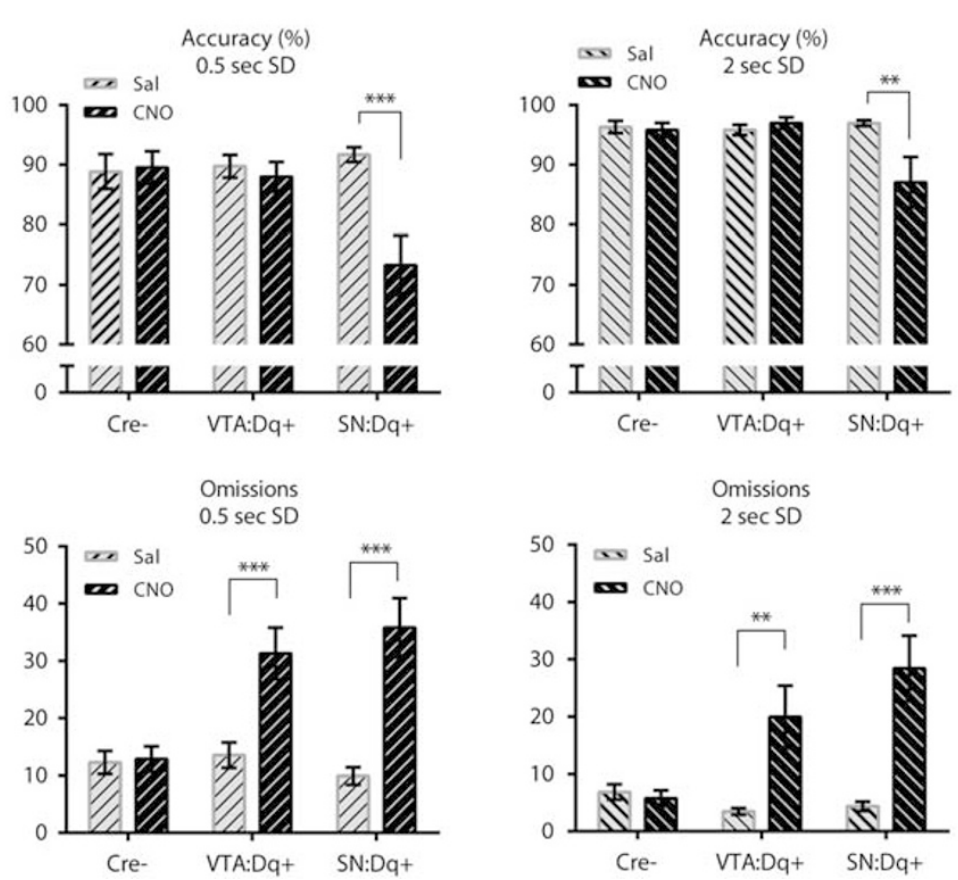

Figure 2 Chemogenetic activation of VTA or SNc DA neurons impairs attentional performance. (a) CNO increased number of omissions in VTA:Dq+ and $\mathrm{SN}: \mathrm{Dq}+$ group. (b) CNO decreased accuracy in SN:Dq+ group. (c) CNO increased latency to make an incorrect response in SN:Dq+ group to $>$ I.0 s (dotted line, represents stimulus duration, SD). (d) Shorter SD decreased accuracy compared with baseline (left panel). CNO decreased accuracy in SN:Dq+ group, also with 0.5 or 2 s SD. (e) Longer SD decreased omissions compared with baseline. CNO increased number of omissions in VTA:Dq+ and SN:Dq+ groups, also with 0.5 or $2 \mathrm{~s} S \mathrm{SD}$. Error bars represent mean $\pm \mathrm{SEM} * P<0.05$, $* * P<0.01$, $* * * *<0.001$.

Table I Effects of Chemogenetic Activation of VTA or SNc DA Neurons on Performance in 5-CSRTT

\begin{tabular}{|c|c|c|c|c|c|c|c|c|c|c|c|c|}
\hline & \multicolumn{4}{|c|}{ Correct (\#) } & \multicolumn{4}{|c|}{ Incorrect (\#) } & \multicolumn{4}{|c|}{ Perseverative responses (\#) } \\
\hline & SAL & SE & CNO & SE & SAL & SE & CNO & SE & SAL & SE & CNO & SE \\
\hline VTA:Dq+ & 88.8 & 2.1 & 68.7 ****** & 4.7 & 5.5 & 1.0 & 3.8 & 1.2 & 23.0 & 7.8 & 14.8 & 6.6 \\
\hline \multirow[t]{2}{*}{$\mathrm{SN}: \mathrm{Dq}^{+}$} & 86.1 & 2.0 & $62.3^{* * * *}$ & 4.5 & 4.8 & 1.0 & $8.6 * *$ & 1.2 & 36.2 & 7.5 & 24.4 & 6.3 \\
\hline & SAL & SE & CNO & SE & SAL & SE & CNO & SE & SAL & SE & CNO & SE \\
\hline Cre- & 0.57 & 0.03 & 0.57 & 0.03 & 0.94 & 0.21 & 0.98 & 0.20 & 1.67 & 0.13 & 1.68 & 0.13 \\
\hline VTA:Dq+ & 0.55 & 0.03 & 0.59 & 0.03 & 1.39 & 0.21 & 0.97 & 0.20 & 1.64 & 0.13 & 1.40** & 0.13 \\
\hline $\mathrm{SN}: \mathrm{Dq}+$ & 0.56 & 0.03 & $0.75 * * * *$ & 0.03 & 1.27 & 0.20 & $1.91 *$ & 0.19 & 1.88 & 0.13 & $2.18^{* * *}$ & 0.13 \\
\hline
\end{tabular}

Figures represent mean $\pm \mathrm{SEM} * P<0.05$, **P $<0.0$ I, **** $P<0.00$ I $\mathrm{CNO}$ compared with saline (SAL). 
post hoc $\mathrm{SN}: \mathrm{Dq}+P<0.0005)$. These attentional deficits were also reflected in a decreased number of correct responses in both VTA:Dq+ and SN:Dq+ groups (Table 1, Group $\times$ Treatment interaction $\mathrm{F}_{2,37}=6.08, P=0.005$, post hoc VTA: $\mathrm{Dq}+$ and $\mathrm{SN}: \mathrm{Dq}+$, both $P<0.0005)$, and an increased number of incorrect responses in the $\mathrm{SN}: \mathrm{Dq}+$ group (Table 1, Group $\times$ Treatment interaction $\mathrm{F}_{2,37}=4.998$, $P=0.012$, post hoc $\mathrm{SN}: \mathrm{Dq}+P=0.003)$. Perseverative responses were not affected by CNO treatment (Table 1, Group $\times$ Treatment interaction $\left.\mathrm{F}_{2,37}=1.818, P=0.177\right)$.

In addition, $\mathrm{CNO}$ treatment affected the rats' response latencies, with differential effects in the VTA:Dq+ and $\mathrm{SN}: \mathrm{Dq}+$ group. $\mathrm{CNO}$ reduced the reward collection latency in VTA:Dq+ rats, whereas this was increased in $\mathrm{SN}: \mathrm{Dq}+$ rats (Table 1, Group $\times$ Treatment interaction $F_{2,37}=10.186$, $P<0.0005$; post hoc VTA:Dq+ $P=0.009$, SN:Dq+ $P=0.001)$. SN:Dq+ rats also showed a delayed latency to make correct or incorrect responses, whereas this was unaffected in VTA:Dq+ rats (Table 1 and Figure 2c, Group $\times$ Treatment interaction correct latency $\mathrm{F}_{2,37}=12.022, \quad P<0.0005$, post hoc SN:Dq+ $P<0.0005$, VTA:Dq $\quad P=0.147$; incorrect latency $\mathrm{F}_{2,37}=3.654$, $P=0.036$, post hoc $\mathrm{SN}: \mathrm{Dq}+P=0.025$, VTA:Dq $+P=0.188)$.

To test whether the attentional deficits caused by $\mathrm{CNO}$ were dependent on attentional load, we tested the animals with a shorter (0.5 s) and longer SD (2 s). Indeed, SD affected the attentional performance: a shorter SD decreased the accuracy (Figure 2d; effect of $\mathrm{SD} \mathrm{F}_{1.57 ; 32.28}=19.603$, $P<0.0005$; post hoc tests $0.5 \mathrm{~s}$ SD vs baseline $P=0.001 ; 2 \mathrm{~s}$ SD $v$ baseline $P=0.069)$, whereas a longer SD decreased omissions (Figure 2e; effect of $\mathrm{SD} \mathrm{F}_{1.81 ; 61,60}=14.819$, $P<0.0005$; post hoc tests $0.5 \mathrm{~s}$ SD vs baseline $P=0.066 ; 2 \mathrm{~s}$ SD vs baseline $P=0.009)$. However, the effect of $\mathrm{CNO}$ in $\mathrm{SN}$ : $\mathrm{Dq}+$ or VTA:Dq+ rats was not affected by these changes in SD. Similar to baseline conditions, CNO decreased accuracy

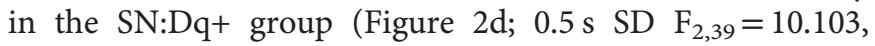
$P<0.0005$, post hoc SN:Dq $P<0.0005 ; 2$ s SD F $2,35=4.935$, $P=0.013$, post hoc $\mathrm{SN}: \mathrm{Dq}+P=0.001)$, and increased omissions in both VTA:Dq+ and $\mathrm{SN}: \mathrm{Dq}+$ groups (Figure 2e; $0.5 \mathrm{~s}$ SD $\mathrm{F}_{2,39}=7.559, P=0.002$, post hoc VTA: $\mathrm{Dq}+$ and $\mathrm{SN}: \mathrm{Dq}+$, both $P<0.0005 ; 2 \mathrm{~s} \mathrm{SD} \mathrm{F}_{2,35}=7.343$, $P=0.002$, post hoc VTA:Dq+ $P=0.002, \quad \mathrm{SN}: \mathrm{Dq}+$ $P<0.0005)$. With saline treatment, one-third $(33 \pm 3.6 \%)$ of omissions occurred following a time-out-as opposed to $67 \%$ following a correct response. Although the percentage omissions made after a time-out was somewhat higher in VTA:Dq+ and SN:Dq+ groups following CNO treatment $(49 \pm 7.2 \%$ and $56 \pm 5.0 \%$, respectively), this difference did not reach statistical significance (Group $\times$ Treatment interaction $\left.\mathrm{F}_{2,36}=2.138, P=0.133\right)$.

In summary, chemogenetic activation of DA neurons in either VTA or SNc significantly impaired attention, which was not dependent on attentional load. In VTA:Dq+ rats, $\mathrm{CNO}$ treatment increased omissions, while in $\mathrm{SN}: \mathrm{Dq}+$ rats, CNO treatment increased omissions and decreased accuracy. Furthermore, activation of VTA DA neurons decreased latency to collect rewards, whereas SNc DA neuron activation increased latency to collect rewards, and to make correct and incorrect responses.

\section{Effects of DA Neuron Activation on Impulsivity}

Under baseline conditions, CNO treatment did not affect the number of premature responses (Figure 3a; Group $\times$ Treatment interaction $\mathrm{F}_{2,37}=2.351, P=0.109$ ), nor the latency to make a premature response (Figure 3b; Group $\times$ Treatment interaction $\left.\mathrm{F}_{2,37}=1.050, P=0.360\right)$.

As a positive control for enhancement of impulsivity, we tested the effects of systemic amphetamine (AMPH) administration, or testing with a longer ITI of $7 \mathrm{~s}$ ( $7 \mathrm{~s}$ ITI). Both manipulations have been reported to increase premature responding in the 5-CSRTT (Baarendse and Vanderschuren, 2012; Cole and Robbins, 1989; Dalley et al, 2007; Van Gaalen et al, 2006). We found that, as expected, both AMPH and a longer ITI increased the number of premature responses (Figure 3c; $0.3 \mathrm{mg} / \mathrm{kg}$ AMPH vs Sal $t_{11}=-2.434, P=0.033 ; 1.0 \mathrm{mg} / \mathrm{kg}$ AMPH $v s$ Sal $t_{11}=-2.603$, $P=0.025 ; 7$ s ITI vs 5 s ITI $t_{12}=-4.951, P<0.0005$; negative control Sal vs Sal $t_{10}=-1.33, P=0.213$ ).

Next, we tested whether CNO would affect impulsive behavior under conditions of increased impulsivity, ie, during a $7 \mathrm{~s}$ ITI, or variable long ITIs, varying from 5 to $15 \mathrm{~s}$ (vITI). We found that longer ITIs profoundly increased the number of premature responses under saline conditions (Figure 3d; main effect of ITI $\mathrm{F}_{2,70}=82.431, P<0.0005$; post hoc comparisons all $P<0.0005)$. With longer ITIs, also the latency to make a premature response was increased (Figure 3e; main effect of ITI $F_{1.7 ; 58.3}=140.446, P<0.0005$; post hoc comparisons all $P<0.0005)$. However, similar to baseline conditions, treatment with $\mathrm{CNO}$ did not affect the number of premature responses (Figure 3d; Group $\times$ Treatment interaction $7 \mathrm{~s}$ ITI: $\mathrm{F}_{2,37}=0.045, \quad P=0.956$; vITI: $\left.\mathrm{F}_{2,39}=0.9, P=0.415\right)$. CNO did significantly decrease the latency to make a premature response, in the VTA:Dq+ group both under $7 \mathrm{~s}$ ITI and vITI conditions, and in the SN: $\mathrm{Dq}+$ group under the vITI condition (Figure 3e; Group $\times$ Treatment interaction $7 \mathrm{~s}$ ITI: $\mathrm{F}_{2,37}=3.778, P=0.032$, post hoc VTA:Dq $+P=0.003$; vITI: $\mathrm{F}_{2,39}=4.872, \quad P=0.013$, post hoc VTA:Dq $+P=0.003, \mathrm{SN}: \mathrm{Dq}+P=0.025)$.

In summary, chemogenetic activation of DA neurons in VTA or SNc did not affect the number of premature responses-either under baseline conditions or during challenges with prolonged ITIs. DA neuron activation did reduce the latency to make a premature response, both in VTA:Dq+ and SN:Dq+ rats, especially during prolonged ITIs.

\section{Dose-Dependent Effects of CNO on Attention}

To determine the dose-dependency of the effects of CNO, we tested five different doses, under baseline conditions ( $5 \mathrm{~s}$ ITI, $1 \mathrm{~s}$ SD). Consistent with our earlier results, we found no significant effect of $\mathrm{CNO}$ on premature responding (Figure 4a; Group $\times$ Treatment interaction $\mathrm{F}_{7.5 ; 135.3}=1.239$, $P=0.284)$. CNO dose-dependently increased omissions in both VTA:Dq+ and SN:Dq+ groups (Figure 4b; Group $\times$ Treatment interaction $\left.\mathrm{F}_{6.0 ; 107.8}=9.551, P<0.0005\right)$. In the $\mathrm{SN}: \mathrm{Dq}+$ group, CNO increased omissions at $0.03 \mathrm{mg} / \mathrm{kg}$ and higher (CNO vs saline $P=0.001 ; 0.1$ and $0.3 \mathrm{mg} / \mathrm{kg}$, both $P<0.001)$; whereas in the VTA:Dq+ group, only the highest dose $(0.3 \mathrm{mg} / \mathrm{kg})$ significantly increased omissions (CNO vs saline $P=0.004)$. In $\mathrm{SN}: \mathrm{Dq}+$ rats, doses of $0.03 \mathrm{mg} / \mathrm{kg}$ and 

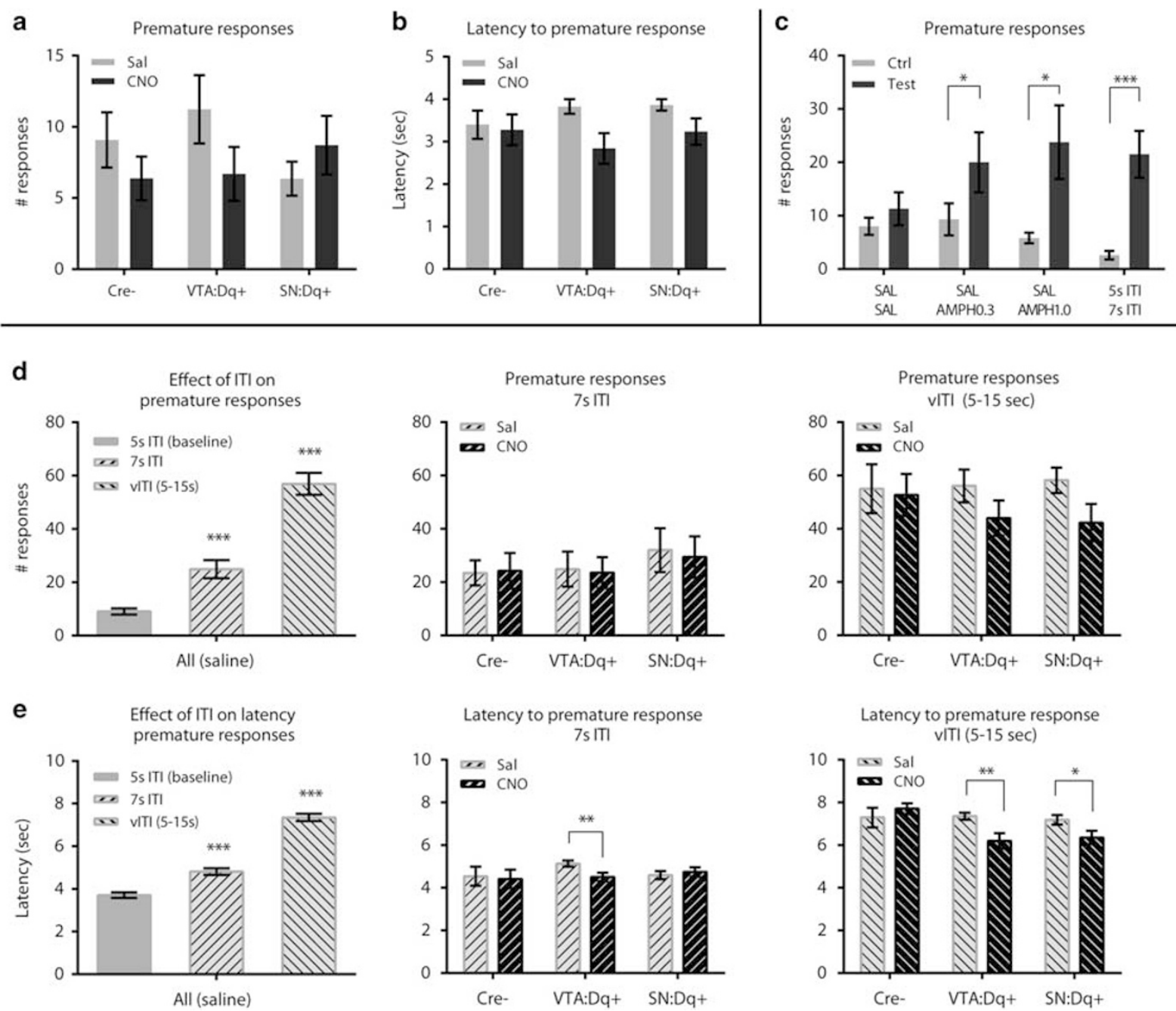

Figure 3 Chemogenetic activation of VTA or SNc DA neurons does not affect premature responses. No significant effect of CNO vs saline on the number of premature responses (a) or latency to make premature response (b). (c) Systemic injections of amphetamine $(0.3$ or $1.0 \mathrm{mg} / \mathrm{kg})$, or prolonged intertrial interval of $7 \mathrm{~s}(7 \mathrm{~s} \mathrm{ITI})$ increased the number of premature responses. (d) Total number of premature responses was increased by challenges with longer ITI (7 s ITI or variable long ITI compared with baseline), but was not affected by CNO. (e) Latency to make premature responses was increased by challenges with longer ITI ( $7 \mathrm{~s}$ ITI or variable long ITI compared with baseline), and decreased by CNO in VTA:Dq+ and SN:Dq+ group. Error bars represent mean \pm SEM * $P<0.05$, *** $P<0.01$, **** $P<0.001$.

higher also decreased accuracy (Figure 4c; Group $\times$ Treatment interaction $\mathrm{F}_{4.6 ; 82.7}=5.515, P<0.0005 ; \mathrm{SN}: \mathrm{Dq}+0.03$, 0.1 and $0.3 \mathrm{mg} / \mathrm{kg} \mathrm{CNO} v s$ saline, all $P<0.01$ ), whilst response latency was increased at 0.1 and $0.3 \mathrm{mg} / \mathrm{kg} \mathrm{CNO}$ (Figure 4d; Group $\times$ Treatment interaction $\mathrm{F}_{4.2 ; 74.8}=5.643$, $P<0.0005 ; \mathrm{SN}: \mathrm{Dq}+0.1$ and $0.3 \mathrm{mg} / \mathrm{kg} \mathrm{CNO} v s$ saline, both $P<0.001)$. No significant effects were found on the latency to collect the reward (Figure 4e; Group $\times$ Treatment interaction $\left.\mathrm{F}_{3.4 ; 61.1}=1.778, \quad P=0.154\right)$ or the latency to make a premature response (Figure 4f; Group $\times$ Treatment interaction $\mathrm{F}_{8 ; 144}=1.311, P=0.242$ ). Thus, low doses of $\mathrm{CNO}$ $(0.03 \mathrm{mg} / \mathrm{kg}$ and higher) were sufficient to impair attention in $\mathrm{SN}: \mathrm{Dq}+$ rats, while only the highest dose tested $(0.3 \mathrm{mg} /$ $\mathrm{kg}$ ) significantly affected attention in VTA:Dq+ rats.

\section{DISCUSSION}

In this study, we show that chemogenetic activation of VTA and SNc DA neurons impairs attentional performance in the
5-CSRTT. In contrast, we found no evidence for increased impulsivity as a result of enhancing midbrain DA neuron activity. In addition, we show that DA neuronal activation in the VTA compared with SNc results in a distinct behavioral profile with respect to attentional accuracy and response latency. Although increasing DA neuron activity in both regions increased omissions, SNc DA neuronal activation decreased accuracy and delayed correct response, incorrect response, and reward collection latency, whereas VTA DA neuronal activation did not affect accuracy, correct response latency, and incorrect response latency, and reduced reward collection latency. Thus, the attentional impairments in these groups were mediated through different mechanisms.

\section{VTA DA Neuronal Activation Impairs Sustained Attention and Enhances Behavioral Responsivity}

We found that chemogenetic activation of DA neurons in the VTA significantly increased omissions in the 5-CSRTT. This 
a

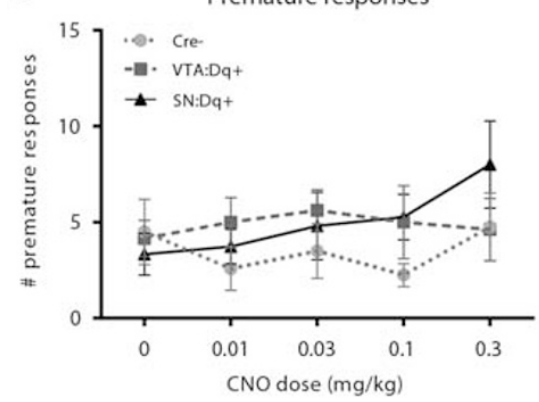

d

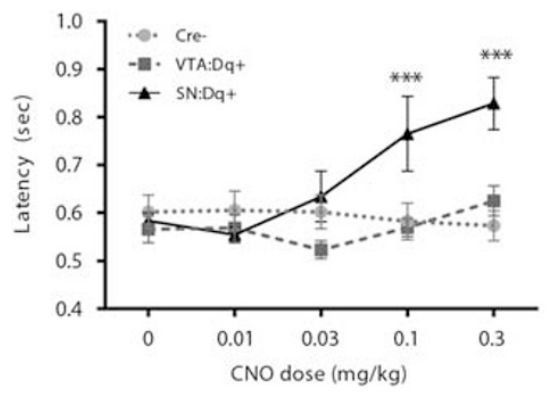

b

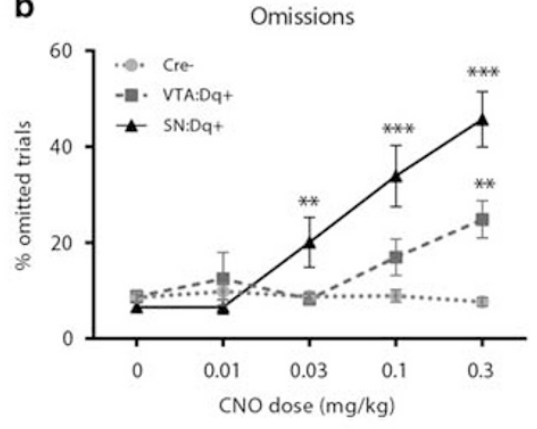

e

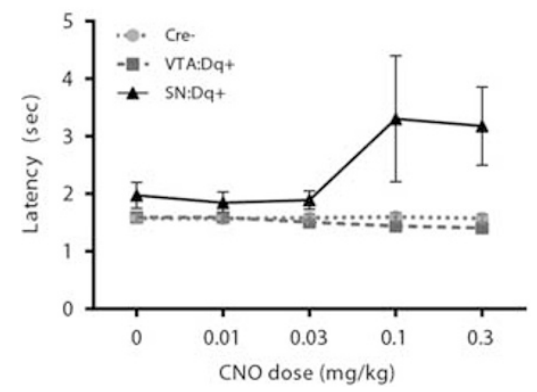

C

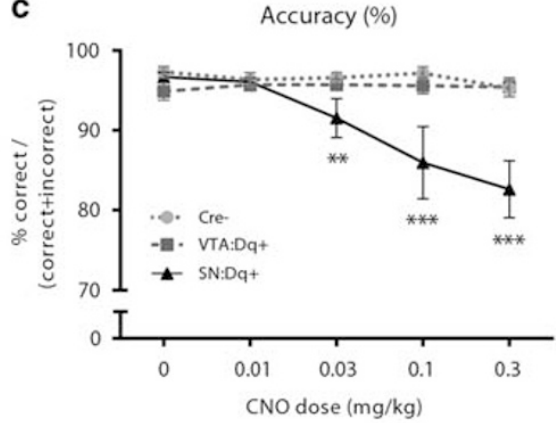

f

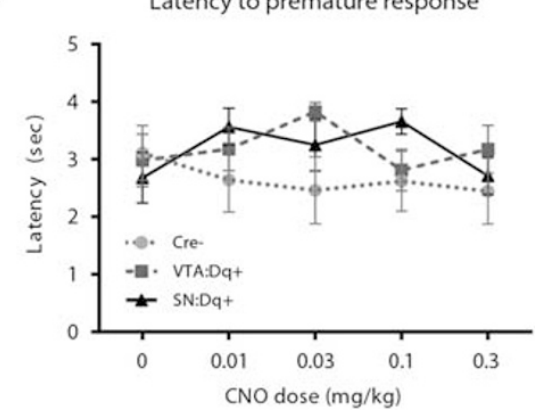

Figure 4 Dose-dependent effects of CNO on 5-CSRTT performance. (a) Premature responses were not affected by CNO. (b) Omissions were increased at $0.03 \mathrm{mg} / \mathrm{kg}$ CNO and higher doses in SN:Dq+ group, and at $0.3 \mathrm{mg} / \mathrm{kg} \mathrm{CNO}$ in VTA:Dq+ group. (c) Accuracy was decreased at $0.03 \mathrm{mg} / \mathrm{kg} \mathrm{CNO}$ and higher doses in SN:Dq+ group, and unaffected in VTA:Dq+ group. (d) Latency to make a correct response was increased at 0.1 and $0.3 \mathrm{mg} / \mathrm{kg} \mathrm{CNO}$ in $\mathrm{SN}: \mathrm{Dq}$ + group, and unaffected in VTA:Dq+ group. (e and f) Latency to collect the reward and latency to make a premature response were not significantly affected by $\mathrm{CNO}$. Error bars represent mean $\pm \mathrm{SEM} * * P<0.0$ I, *** $P<0.001 \mathrm{CNO}$ compared with saline $(0 \mathrm{mg} / \mathrm{kg} \mathrm{CNO})$.

effect persisted during tests with a variable attentional load as a result of shorter or longer SDs. Furthermore, VTA DA neuron activation decreased the latency to collect rewards and to make premature responses, whereas the attentional accuracy was unaffected. These results are in line with a role for mesolimbic DA in the speed and probability of responding, but not in attentional accuracy (Cole and Robbins, 1989). In the present study, we have focused on the role of midbrain DA neuronal activity, whereas the majority of previous studies has investigated DA signaling in striatal and cortical target areas. On the basis of our immunohistochemical results, we presume that VTA DA neuron activation primarily resulted in enhanced DA signaling in the ventral and dorsomedial parts of the striatum. We expect a relatively small contribution of altered mesocortical DA signaling to the observed behavioral effects, as DA levels have been shown to be significantly lower in PFC compared with striatum (10-20-fold difference; Ihalainen et al, 1999; Moghaddam et al, 1990), and we were not able to detect DREADD expression in the PFC. However, future studies should clarify the neurochemical effects of chemogenetic DA neuron activation in target regions of VTA DA neurons. Previous pharmacological studies have shown that infusions of DA-R antagonists or partial agonists into NAc or DMS increased omissions (Besson et al, 2010; Pezze et al, 2007), whereas local DA stimulation in NAc Core and Shell sub-regions did not affect attention in the 5-CSRTT (Economidou et al, 2012; Moreno et al, 2013). In addition, enhanced DA signaling in the medial PFC (mPFC), through reuptake inhibition or D1-R agonism, has been shown to decrease omissions (Agnoli and Carli, 2011; Economidou et al, 2012; Granon et al, 2000). These results are, to some extent, in contrast with our observation that enhanced activity of mesocorticolimbic DA neurons increased the number of omitted trials. However, systemic administration of a D1- or D2-R agonist was shown to have the same effect (Winstanley et al, 2010). Importantly, to fully understand the contribution of DA signaling to omissions in the 5-CSRTT, it is essential to consider the broader behavioral phenotype and its underlying mechanisms. For example, treatment with selective D1- or D2-R antagonists may impair stimulus discrimination, resulting in more omissions as well as decreased accuracy (Pezze et al, 2007). In contrast, enhanced VTA DA neuronal activity may affect other functions, such as behavioral activation (see below), thereby affecting omissions and response latency, while leaving accuracy intact. Future studies are needed to further distinguish the neurochemical and functional mechanisms that determine the effects of DA signaling on attentional behavior.

DA signaling in the NAc has been implicated in approach initiation, especially in anticipation of a reward (Hamid et al, 2015; Ko and Wanat, 2016; Nicola, 2010; Syed et al, 2015). We found that VTA DA neuronal activation reduced the rats' latency to retrieve rewards, and to make premature responses. In contrast, the latency to make correct or incorrect responses was unaffected. This suggests that, at least under some conditions, enhanced VTA DA neuronal activity promotes rapid action initiation. A fast response initiation may be interpreted as enhanced behavioral activation and/or increased motivation, processes that both involve mesolimbic DA (Nicola, 2010; Robbins and Everitt, 2007; Salamone and Correa, 2012; Wise, 2004). Previously, we have shown that chemogenetic activation of VTA mesolimbic neurons, or local D2-R knockdown, increased 
motivation to work for sucrose in rats (Boender et al, 2014; de Jong et al, 2015). However, in the present study, we showed that VTA DA neuron activation increased omissions, and did not affect latency to make a correct nose poke, which contradicts a putative increase in motivation. Therefore, we propose that the behavioral profile, including more omissions and faster responses, may reflect effects on behavioral activation rather than motivation. Indeed, chemogenetic activation of midbrain DA neurons or VTA to NAc pathway has been shown to increase locomotor activity (Boekhoudt et al, 2016; Boender et al, 2014; Wang et al, 2013), consistent with a role for increased mesolimbic DA activity in locomotor hyperactivity (Ikemoto, 2002; Kelly et al, 1975). As a result, increased VTA DA neuronal activity likely interferes with the animal's ability to focus attention on a specific stimulus during a restricted time period, thus impairing sustained attention. Enhanced responsivity to cues may have a dual effect on task performance in the 5-CSRTT. On one hand, it may facilitate the initiation of certain actions, resulting in faster responses. On the other hand, locomotor hyperactivity and responsivity to environmental stimuli that are not relevant for task performance would distract the animal's attention away from the cue lights, and result in more omissions.

\section{SNc DA Neuronal Activation Impairs Attention and Delays Responsivity}

Chemogenetic activation of SNc DA neuronal activity caused significant attentional deficits in the 5-CSRTT, as apparent from increased omissions and reduced accuracy. In addition, response latencies were increased, ie, the rats were slower to make nose-poke responses and to collect the reward. Interestingly, similar results have been observed following lesions of the DMS or DLS, or dorsal striatal DA depletion (Baunez and Robbins, 1999; Rogers et al, 2001). These findings indicate that the nigrostriatal DA pathway is crucially involved in attentional processes, and that disruption of this pathway, either by inhibition or stimulation, may severely affect behavioral performance.

The dorsal striatum is part of a neural circuitry involving frontal cortex and basal ganglia, that regulates divided attention and executive function (Alexander et al, 1986; Dalley et al, 2008; Wimmer et al, 2015). Within this circuitry, the dorsal striatum serves an important role in sensorimotor integration and response control (Nieoullon, 2002; Rogers et al, 2001). The DMS receives projections from the PFC, including medial PFC and anterior cingulate cortex (ACC) (Donoghue and Herkenham, 1986; McGeorge and Faull, 1989). Importantly, lesions or chemogenetic inhibition of these cortical regions have been shown to impair attentional accuracy and response latencies in the 5-CSRTT, similar to the effects of nigrostriatal DA dysfunction (Chudasama et al, 2003; Koike et al, 2015; Muir et al, 1996). In addition, glutamatergic and DAergic signaling in this cortico-striatal circuitry have been shown to interact in the regulation of attentional performance (Agnoli and Carli, 2011; Agnoli et $a l, 2013)$. Our results complement these findings by showing that enhanced DA neuronal activity in the SNc also results in impaired attention and response control, comparable to dorsal striatal or prefrontal dysfunction. Previously, it was shown that a selective D1-R agonist or antagonist infused into $\mathrm{mPFC}$ could improve or impair attentional accuracy in rats, respectively, depending on low or high baseline performance (Granon et al, 2000). Taken together, these results suggest that there is an optimal level of DA signaling required for accurate task performance. Here, we show a novel finding, indicating that also enhanced DA signaling in the nigrostriatal pathway can be detrimental for attentional performance. We found that SNc DA neuronal activation consistently increased omissions, impaired accuracy, and delayed response latency, irrespective of attentional load. An increase in omissions and response latencies could indicate a reduced motivational state or impaired motor function. However, activation of SNc DA neurons with a low dose of $\mathrm{CNO}$, that did not affect response latencies, was sufficient to impair attention. Furthermore, optogenetic activation of SNc DA neurons has been shown to promote voluntary movements (Barter et al, 2015). Together, this indicates that enhanced SNc DA neuronal activity is sufficient to impair attentional performance, without detrimental effects on motor function or motivation. On the basis of behavioral deficits in patients with Parkinson's disease, it has been suggested that nigrostriatal dysfunction affects the selection of an appropriate motor response, in particular, when there is uncertainty about the stimulus and its associated response (Nieoullon, 2002). Again, our data suggest that enhanced nigrostriatal DA activity results in a similar phenotype: the animals appear to have difficulty selecting the appropriate response to an unpredictable stimulus, which may result in a slower response, selection of an incorrect motor response, or an omission. We suggest that enhanced SNc DA neuronal activity disrupts DA signaling in dorsal striatal regions, and thereby interferes with sensorimotor integration and the control of appropriate responses to environmental stimuli. However, relatively little is known about the effects of increased nigrostriatal DA activity, and future studies need to elucidate which anatomical and neurochemical aspects are causally related to the observed attentional impairments.

\section{Midbrain DA Neuronal Activation does not Induce Impulsivity}

Chemogenetic activation of DA neurons in either the VTA or SNc did not affect impulsivity in the 5-CSRTT. Previous studies have implicated DA in the control of impulsive actions, particularly DA signaling in the (ventral and dorsomedial) striatum (Agnoli et al, 2013; Cole and Robbins, 1989; Economidou et al, 2012; Moreno et al, 2013; Pattij et al, 2007; Pezze et al, 2007). Therefore, we hypothesized that enhancing midbrain DA neuronal activity, in particular of VTA DA neurons that project to NAc or DMS, would increase impulsivity. The discrepancy of our findings with previous reports may be related to several factors, including anatomical sub-region, magnitude of manipulation, and individual differences in impulsivity (Economidou et al, 2012; Moreno et al, 2013; Pezze et al, 2007; Winstanley et al, 2010). Importantly, the effects of CNO depend on endogenous neuronal activity. As mentioned above, neurochemical studies have indicated that phasic DA release in the NAc-related to VTA DA neuron burst firing-is particularly associated with action initiation in anticipation of a reward (Hamid et al, 2015; Roitman, 2004; Syed et al, 2015). This typically occurs in 
response to a cue that is associated with a reward, such as a light stimulus. When postsynaptic DA signaling in the NAc is pharmacologically elevated, eg, by amphetamine or DA-R agonists, this may mimic the neurochemical situation of 'action initiation in anticipation of a reward', and thus the animal is driven to make a response. During the ITI, this would result in a premature response. Indeed, consistent with previous findings (Baarendse and Vanderschuren, 2012; Cole and Robbins, 1989; Van Gaalen et al, 2006), we observed that systemic administration of amphetamine (inducing DA release at synaptic terminals) increased the number of premature responses, and previous studies showed that infusion of a DA-R agonist or a reuptake inhibitor into the NAc or DMS does the same (Agnoli et al, 2013; Moreno et al, 2013; Pezze et al, 2007). CNO, however, is expected to preferentially enhance DA activity when DA neurons are already endogenously active. During the ITI, when the animals are waiting and no particular cues are presented that predict reward availability, there is presumably low baseline DA neuronal activity (Hamid et al, 2015; Syed et al, 2015), although there is evidence for 'ramping' activity in anticipation of the stimulus in VTA DA target areas (Donnelly et al, 2015). When the waiting period is longer than expected (prolonged ITI), this may drive the animal to make a response before stimulus onset. We found that, under these high-impulsive conditions, CNO reduced the latency to perform premature responses in both VTA:Dq+ and SN:Dq+ groups, but the total number of premature responses remained unaffected. Our results therefore indicate that enhancing endogenous DA neuron activity in either VTA or $\mathrm{SNc}$ may promote action initiation vigor, but is in itself not sufficient to induce impulsive actions.

\section{SUMMARY AND CONCLUSION}

In this study, we determined the effects of enhanced DA neuronal activity in VTA and SNc on attentional performance and impulsive behavior in rats. We found that VTA DA neuronal activation promotes the vigor of goal-directed actions, but also impairs sustained attention. These effects likely result from enhanced behavioral responsivity. On the other hand, SNc DA neuronal activation impaired sustained attention and accuracy, and slowed responsiveness in the task. This may be attributed to disrupted sensorimotor integration, resulting in impaired execution of a stimulus-induced response. Thus, DA neurons in the VTA or SNc support distinct aspects of cognitive performance. Our findings contribute to the understanding of the neural substrates underlying attention deficits and impulsivity, and provide valuable insights to improve treatment of these symptoms in the clinic.

\section{FUNDING AND DISCLOSURE}

This project was funded by NeuroBasic, Nudge-IT (FP7KBBE.2013.2.2-01), and ZonMW. The authors declare no conflict of interest.

\section{ACKNOWLEDGMENTS}

We kindly thank Bryan Roth and the NIMH Chemical Synthesis and Drug Supply Program for providing CNO, and Rixt van der Veen for sharing her MedPC scripts.

\section{REFERENCES}

Agnoli L, Carli M (2011). Synergistic interaction of dopamine D1 and glutamate N-methyl-d-aspartate receptors in the rat dorsal striatum controls attention. Neuroscience 185: 39-49.

Agnoli L, Mainolfi P, Invernizzi RW, Carli M (2013). Dopamine D1-like and D2-like receptors in the dorsal striatum control different aspects of attentional performance in the five-choice serial reaction time task under a condition of increased activity of corticostriatal inputs. Neuropsychopharmacology 38: 701-714.

Alexander GE, Delong MR, Strick PL (1986). Parallel organization of functionally segregated circuits linking basal ganglia and cortex. Annu Rev Neurosci 9: 357-381.

American Psychiatric Association (2013). Diagnostic and statistical manual of mental disorders. American Psychiatric Association: Washington, DC, USA.

Armbruster BN, Li X, Pausch MH, Herlitze S, Roth BL (2007). Evolving the lock to fit the key to create a family of $G$ proteincoupled receptors potently activated by an inert ligand. Proc Natl Acad Sci USA 104: 5163-5168.

Baarendse PJJ, Vanderschuren LJMJ (2012). Dissociable effects of monoamine reuptake inhibitors on distinct forms of impulsive behavior in rats. Psychopharmacology (Berl) 219: 313-326.

Bari A, Robbins TW (2013). Inhibition and impulsivity: behavioral and neural basis of response control. Prog Neurobiol 108: 44-79.

Barter JW, Li S, Lu D, Bartholomew R a., Rossi M a., Shoemaker CT et al (2015). Beyond reward prediction errors: the role of dopamine in movement kinematics. Front Integr Neurosci 9: $1-22$.

Baunez C, Robbins TW (1999). Effects of dopamine depletion of the dorsal striatum and further interaction with subthalamic nucleus lesions in an attentional task in the rat. Neuroscience 92: 1343-1356.

Besson M, Belin D, McNamara R, Theobald DE, Castel A, Beckett VL et al (2010). Dissociable control of impulsivity in rats by dopamine $\mathrm{d} 2 / 3$ receptors in the core and shell subregions of the nucleus accumbens. Neuropsychopharmacology 35: $560-569$.

Bjorklund A, Dunnett SB (2007). Dopamine neuron systems in the brain: an update. Trends Neurosci 30: 194-202.

Boekhoudt L, Omrani A, Luijendijk MCM, Wolterink-Donselaar IG, Wijbrans EC, van der Plasse G et al (2016). Chemogenetic activation of dopamine neurons in the ventral tegmental area, but not substantia nigra, induces hyperactivity in rats. Eur Neuropsychopharmacol 26: 1784-1793.

Boender AJ, de Jong JW, Boekhoudt L, Luijendijk MCM, van der Plasse G, Adan RAH (2014). Combined use of the canine adenovirus-2 and DREADD-technology to activate specific neural pathways in vivo. PLoS One 9: e95392.

Carli M, Robbins TW, Evenden JL, Everitt BJ (1983). Effects of lesions to ascending noradrenergic neurones on performance of a 5 -choice serial reaction task in rats; implications for theories of dorsal noradrenergic bundle function based on selective attention and arousal. Behav Brain Res 9: 361-380.

Chudasama Y, Passetti F, Rhodes SE V, Lopian D, Desai A, Robbins TW (2003). Dissociable aspects of performance on the 5choice serial reaction time task following lesions of the dorsal anterior cingulate, infralimbic and orbitofrontal cortex in the rat: Differential effects on selectivity, impulsivity and compulsivity. Behav Brain Res 146: 105-119.

Cole BJ, Robbins TW (1989). Effects of 6-hydroxydopamine lesions of the nucleus accumbens septi on performance of a 5-choice serial reaction time task in rats: Implications for theories of selective attention and arousal. Behav Brain Res 33: 165-179.

Cools R (2011). Dopaminergic control of the striatum for high-level cognition. Curr Opin Neurobiol 21: 402-407.

Dalley JW, Everitt BJ, Robbins TW (2011). Impulsivity, compulsivity, and top-down cognitive control. Neuron 69: 680-694. 
Dalley JW, Laane K, Theobald DE, Pena Y, Bruce CC, Huszar AC et al (2007). Enduring deficits in sustained visual attention during withdrawal of intravenous methylenedioxymethamphetamine self-administration in rats: results from a comparative study with d-amphetamine and methamphetamine. Neuropsychopharmacology 32: 1195-1206.

Dalley JW, Mar AC, Economidou D, Robbins TW (2008). Neurobehavioral mechanisms of impulsivity: fronto-striatal systems and functional neurochemistry. Pharmacol Biochem Behav 90: 250-260.

de Jong JW, Roelofs TJM, Mol FMU, Hillen AEJ, Meijboom KE, Luijendijk MCM et al (2015). Reducing ventral tegmental dopamine D2 receptor expression selectively boosts incentive motivation. Neuropsychopharmacology 40: 2085-2095.

Donnelly NA, Paulsen O, Robbins TW, Dalley JW (2015). Ramping single unit activity in the medial prefrontal cortex and ventral striatum reflects the onset of waiting but not imminent impulsive actions. Eur J Neurosci 41: 1524-1537.

Donoghue JP, Herkenham M (1986). Neostriatal projections from individual cortical fields conform to histochemically distinct striatal compartments in the rat. Brain Res 365: 397-403.

Eagle DM, Baunez C (2010). Is there an inhibitory-response-control system in the rat? Evidence from anatomical and pharmacological studies of behavioral inhibition. Neurosci Biobehav Rev 34: 50-72.

Economidou D, Theobald DE, Robbins TW, Everitt BJ, Dalley JW (2012). Norepinephrine and dopamine modulate impulsivity on the five-choice serial reaction time task through opponent actions in the shell and core sub-regions of the nucleus accumbens. Neuropsychopharmacology 37: 2057-2066.

Everitt BJ, Robbins TW (2005). Neural systems of reinforcement for drug addiction: from actions to habits to compulsion. Nat Neurosci 8: 1481-1489.

Franken IH a, Booij J, Van Den Brink W (2005). The role of dopamine in human addiction: from reward to motivated attention. Eur J Pharmacol 526: 199-206.

Van Gaalen MM, Brueggeman RJ, Bronius PFC, Schoffelmeer ANM, Vanderschuren LJMJ (2006). Behavioral disinhibition requires dopamine receptor activation. Psychopharmacology (Berl) 187: 73-85.

Granon S, Passetti F, Thomas KL, Dalley JW, Everitt BJ, Robbins TW (2000). Enhanced and impaired attentional performance after infusion of D1 dopaminergic receptor agents into rat prefrontal cortex. J Neurosci 20: 1208-1215.

Hamid AA, Pettibone JR, Mabrouk OS, Hetrick VL, Schmidt R, Vander Weele CM et al (2015). Mesolimbic dopamine signals the value of work. Nat Neurosci 19: 117-126.

Howes OD, Kapur S (2009). The dopamine hypothesis of schizophrenia: version III-the final common pathway. Schizophr Bull 35: 549-562.

Ihalainen JA, Riekkinen P, Feenstra MGP (1999). Comparison of dopamine and noradrenaline release in mouse prefrontal cortex, striatum and hippocampus using microdialysis. Neurosci Lett 277: $71-74$.

Ikemoto S (2002). Ventral striatal anatomy of locomotor activity induced by cocaine, D-amphetamine, dopamine and D1/D2 agonists. Neuroscience 113: 939-955.

Kelly PH, Seviour PW, Iversen SD (1975). Amphetamine and apomorphine responses in the rat following 6-OHDA lesions of the nucleus accumbens septi and corpus striatum. Brain Res 94: 507-522.

Ko D, Wanat MJ (2016). Phasic dopamine transmission reflects initiation vigor and exerted effort in an action- and regionspecific manner. J Neurosci 36: 2202-2211.

Koike H, Demars MP, Short JA, Nabel EM, Akbarian S, Baxter MG et al (2015). Chemogenetic inactivation of dorsal anterior cingulate cortex neurons disrupts attentional behavior in mouse. Neuropsychopharmacology 41: 1-40.
McGeorge AJ, Faull RLM (1989). The organization of the projection from the cerebral cortex to the striatum in the rat. Neuroscience 29: 503-537.

Moghaddam B, Roth RH, Bunney BS (1990). Characterization of dopamine release in the rat medial prefrontal cortex as assessed by in vivo microdialysis: comparison to the striatum. Neuroscience 36: 669-676.

Moore Y, Bloom E (1978). Central catecholamine neuron systems : anatomy. Annu Rev Neurosci 1: 129-169.

Moreno M, Economidou D, Mar A. C, López-Granero C, Caprioli D, Theobald DE et al (2013). Divergent effects of D2/3 receptor activation in the nucleus accumbens core and shell on impulsivity and locomotor activity in high and low impulsive rats. Psychopharmacology (Berl) 228: 19-30.

Muir JL, Everitt BJ, Robbins TW (1996). The cerebral cortex of the rat and visual attentional function: dissociable effects of mediofrontal, cingulate, anterior dorsolateral, and parietal cortex lesions on a five-choice serial reaction time task. Cereb Cortex 6: 470-481.

Nicola SM (2010). The flexible approach hypothesis: unification of effort and cue-responding hypotheses for the role of nucleus accumbens dopamine in the activation of reward-seeking behavior. J Neurosci 30: 16585-16600.

Nieoullon A (2002). Dopamine and the regulation of cognition and attention. Prog Neurobiol 67: 53-83.

Pattij T, Janssen MCW, Vanderschuren LJMJ, Schoffelmeer ANM, Van Gaalen MM (2007). Involvement of dopamine D1 and D2 receptors in the nucleus accumbens core and shell in inhibitory response control. Psychopharmacology (Berl) 191: 587-598.

Pattij T, Vanderschuren LJMJ (2008). The neuropharmacology of impulsive behaviour. Trends Pharmacol Sci 29: 192-199.

Pezze M-A, Dalley JW, Robbins TW (2007). Differential roles of dopamine D1 and D2 receptors in the nucleus accumbens in attentional performance on the five-choice serial reaction time task. Neuropsychopharmacology 32: 273-283.

Robbins TW, Everitt BJ (2007). A role for mesencephalic dopamine in activation: commentary on Berridge (2006). Psychopharmacology (Berl) 191: 433-437.

Rogers RD, Baunez C, Everitt BJ, Robbins TW (2001). Lesions of medial and lateral striatum in the rat produce differential deficits in attentional performance. Behav Neurosci 115: 799-811.

Roitman MF (2004). Dopamine operates as a subsecond modulator of food seeking. J Neurosci 24: 1265-1271.

Salamone JD, Correa M (2012). The mysterious motivational functions of mesolimbic dopamine. Neuron 76: 470-485.

Syed ECJ, Grima LL, Magill PJ, Bogacz R, Brown P, Walton ME (2016). Action initiation shapes mesolimbic dopamine encoding of future rewards. Nat Neurosci 19: 34-36.

Tripp G, Wickens JR (2009). Neurobiology of ADHD. Neuropharmacology 57: 579-589.

Volkow ND, Baler RD (2015). NOW vs LATER brain circuits: implications for obesity and addiction. Trends Neurosci 38: 345-352.

Wang S, Tan Y, Zhang J-E, Luo M (2013). Pharmacogenetic activation of midbrain dopaminergic neurons induces hyperactivity. Neurosci Bull 29: 517-524.

Wimmer RD, Schmitt LI, Davidson TJ, Nakajima M, Deisseroth K, Halassa MM (2015). Thalamic control of sensory selection in divided attention. Nature 526: 705-709.

Winstanley CA, Zeeb FD, Bedard A, Fu K, Lai B, Steele C et al (2010). Dopaminergic modulation of the orbitofrontal cortex affects attention, motivation and impulsive responding in rats performing the five-choice serial reaction time task. Behav Brain Res 210: 263-272.

Wise R a (2004). Dopamine, learning and motivation. Nat Rev Neurosci 5: 483-494. 